\title{
Software development firmware system for broken rotor bar detection and diagnosis of induction motor through current signature analysis
}

\author{
H. Pita ${ }^{1}$, G. Zurita ${ }^{1}$ and A. Villarroel ${ }^{1}$ \\ ${ }^{1}$ Laboratory of Industrial Innovation Technology and Robotics (LITIR), Faculty of Engineering and Architecture, Universidad Privada Boliviana \\ (UPB), Cochabamba, Bolivia.
}

ABSTRACT - The induction motors (IMs) are undoubtedly the most used machines in industries because of the advantages they offer such as simplicity, service continuity and low cost. Due to wear and tear, the motor suffers different types of mechanical and electrical failures. Depending on the criticality of the plant motors, it could be necessary to implement predictive techniques in order to detect the faults before they can cause unnecessary downtime. Therefore, in this paper, the research approach was to develop a low cost measurement system based on a micro controller platform for machine diagnosis. The FRDM K64F developing board was selected as the most suitable for satisfying the system conditions, and it was used to collect induction motor's current data. In order to validate the accuracy of the developed system, the Frequency Transfer Functions (FRF) of the developed measurement system and the standard system (NI USB-6009) were compare. It showed a flat frequency spectrum from 0 to $1 \mathrm{KHz}$, with small fluctuations of about 0.25 $\mathrm{dB}$ standard deviation. A fully automated test bench was implemented, which allows to perform all the measurement tests with the IMs, and in this case, the detection and diagnosis of broken bars. Around 240 tests were performed with varying loads, different rotation speeds, and with different severity damage levels in the rotor. The data analysis procedure for broken rotor bar detection and motor diagnosis was performed by the Motor Current Signature Analysis (MCSA), FFT and Enveloped Analysis (EA). Finally, the research approach was successfully accomplished, by the team by developing a software firmware measurement ultra-low cost development platform for machine diagnosis. It was also developed a proper antialiasing filter to reduce industrial noise. The effectiveness of the proposed system is detecting a weak fault in a noise signal. It was found out a new consistent and robust parameter called the pole pass frequency $\left(f_{p s f}\right)$, which could be used as a diagnosis parameter for detection of broken rotor bars faults, with their damage severity degree. The detected parameter can be found around $2.6 \mathrm{~Hz}$, and it increases in amplitude with increasing damage severity.
ARTICLE HISTORY

Revised: $6^{\text {th }}$ May 2020

Accepted: $31^{\text {st }}$ May 2020

\section{KEYWORDS}

Motor of current signature analysis;

test bench;

fast fourier transform; envelope analysis; broken rotor bars; firmware.

\section{INTRODUCTION}

Induction motors (IMs) are considered the most widely used electrical drive system in the industry, due to reliability, service continuity, low cost, robustness and easy maintenance. It is considered the contribution around $85 \%$ of installed induction motors in the industry [1]. The increasing demand on IMs performance and effectiveness, necessitates, a proper machine diagnostic and a systematic preventive maintenance to increase machine efficiency, and reduce downtimes. Moreover, the broken rotor bars denote up to $10 \%$ of motor faults. The motor faults can happen without any warning signs, and it can propagate to next bars leading sudden shutdown of IMs.

The current trends of reducing costs, heading to higher quality production and preventing interruptions in production processes, have made increasingly indispensable to monitor and control the internal operation of the machines and thus detect unexpected signs of failure. A maintenance methodology which is working very well in industry, is the condition based maintenance (CBM). It is based on the maintenance program and recommends decisions based on data continuously recorded of the condition monitoring of the machine [2 - 4].

Based on the literature review, the induction motor fault diagnosis developed in recent years, associated with MCSA. There are two main types of analysis techniques to diagnose broken rotor bars, invasive and non-invasive fault diagnosis technique [5]. The first one performs the diagnosis with costly measurement equipment and expensive sensors (magnetic potential sensor, gyration radius, torque and tachometer). Our research intention was to develop a data acquisition system, a non-invasive fault diagnosis technique with low cost.

The MCSA was originally developed by "Oak Ridge National Laboratory" for the diagnosis of electric motors [6]. The MCSA method was applied to diagnose wear and tear in a motor that operated valves at a nuclear plant. Thereafter, it was effectively applied in the diagnosis of faults in different types of motors that drive other devices such as air conditioning systems, pumps, and blowers. The MCSA showed some limitations for fault detection, particularly, for 
incipient faults [1]. Therefore, Ferñao et al. [7] applied MCSA with the power spectrum analysis to obtain more information from IMs. Vishwanath et al. [8] developed methods with MCSA and vibration analysis for diagnosis for IMs to detect the air gap eccentricity using non-invasive sensors. Roque et al. [9] applied the vibration analysis and MCSA for monitory conditions in IMs to obtain encouragingly results with both methods. Dlamini et al. [10] applied signal vibration signatures to optimize the IMs efficiency with non-invasive sensors. In order to reduce limitations of the MCSA to be use in machine diagnosis. The work done by Kumar et al. [11] opens a wide range of possibilities to use advanced digital signal processing to detect faults and artificial intelligence for fault classification. They applied artificial intelligence for a learning vector quantize neural network based for fault classification and optimization of IMs. Eren et al. [12] developed a four FIR filters bank and implemented a FPGA for reduction of the overall computation time for Motor Current Signature Analysis. The signal processing technique and the Hilbert transform were used for further improved in Mina et al. [13] for fault detection. Aouabdi et al. [14] applied the methodology of MCSA for detection, successfully, gear tooth pitting defects by using multi-scale entropy and principal components analysis. Both the approach and results were further improved by using digital signal processing and wavelets. In order to improve further the digital signal processing approach, Bravo et al. [15] applied the MCSA, wavelets and dual time synchronous averaging for condition monitoring with successful results in gearboxes for industrial environment. Wavelets were used to handle the non-stationary signal, due to FFT has some limitation for that type of signals. The envelope analysis was used to enhance the area of interest.

There are a quite limited number of published papers regarding development of data acquisition data system. Therefore, below are references of the papers related to designing and developing of vibration analysis or other devices. Bilski et al. [16] designed and developed a low-cost real-time effective spectrum analyzer. The major impact was the approach in creating low-cost equipment with the functionality of more complex devices. Adeyeri et al. [17] developed a system using temperature and vibration maintenance models integrating concepts for machine monitoring. Zhang et al. [18] introduced a DAQ system based on a 32-bit STM32F103VET6 microcontroller for rotating machinery monitoring. A three axis accelerometer to measure vibration signals were applied. Huang et al. [19] designed a vibration signal acquisition and analysis system. It was implemented on a STM32 microcontroller, independent ADC, and Wi-Fi modules. Hester et al. [20] presented a wireless sensor network to perform vibration-based structural health monitoring on historic monuments. They designed their own custom sensor board using Eagle (Autodesk, San Rafael, CA, USA). The sensor includes a low noise 1521L accelerometer (Silicon Designs, Kirkland, WA, USA), a solar panel for solar harvesting, a MSP430 launch pad (Texas Instruments) for processing, and a CC2500 2.4GHz radio transceiver (Texas Instruments) for wireless communication. Herrasti et al. [21] introduced an autonomous sensor node that measures acceleration, strain, and temperature. The node uses a three layer printed circuit board (PCB) which is powered by a small Lithium Polymer battery. The signal conditioner module consists of an EFM32 low power microcontroller (Silicon Labs, Austin, TX, USA). Our research group, A. Villarroel et al. [22] designed and developed a low-cost measurements system for machine diagnosis based on vibration analysis. The FRDM K64F development board was chosen as the most suitable for satisfying the system requirements. The industrial environment is highly contaminated by noise a proper antialiasing filter to reduce industrial noise was also implemented. In order to validate the developed system, calculations of frequency response were carried out.

The literature survey reveals there is no scientific publications regarding designing and developing a measurement system to handle induction motor's current data for fault detection and machine diagnosis. Therefore, to mind the research gap, in this paper, a low cost induction motor`s current measurement system based on a microcontroller development board is presented. Thus, in order to perform measurements, a lab bench was designed and implemented allowing several fault simulations. The Motor Current Signature Analysis Method (MCSA), FFT and Envelope Analysis (EA) were used to detect fault and diagnosis in induction motors.

According to statistics, a proper machine maintenance can save $30 \%$ of the total maintenance costs. The Conditions Based Maintenance (CBM), decisions are based on data collected through condition monitoring [2, 23-25]. One common type of condition monitoring is Motor Current Signature Analysis (MCSA). Unfortunately, the equipment required to perform data recording can be costly and unaffordable for smaller companies, especially in developing countries.

The outline of this paper is organized as follows: Section of materials and methods embrace the theoretical background of three phase induction motors and variable frequency drive (VFD). It contains also the fundamentals of MCSA and advanced signal processing signals (FFT \& EA). The development of Firmware software for MCSA procedure to build up a low cost measurement system was also presented. Thereafter, the measurement was set-up and procedures were incorporated. Finally, the paper presents results, discussions, and conclusions.

\section{MATERIALS AND METHODS}

\section{Three Phase Induction Motor}

A three phase induction motor is an AC electric motor in which the electric current in the rotor needed to produce the torque is obtained by the electromagnetic induction from the magnetic field of the stator windings. There are two types of induction motors: squirrel-cage rotor and wound type rotor. The most common used in the industry is the first one due to reliability, economy and rugged type. The wound type rotor induction machine, has windings on both the stator and 
the rotor, housed in respective slots, meanwhile the squirrel-cage type rotor has several bars joined in both ends with rings.

Figure 1 illustrates an asynchronous motor with 6 grooves in the stator and it is a squirrel-cage type, three-phase induction motor, in which each phase is constructed with a coil displaced $120^{\circ}$ with respect to the other two [26].

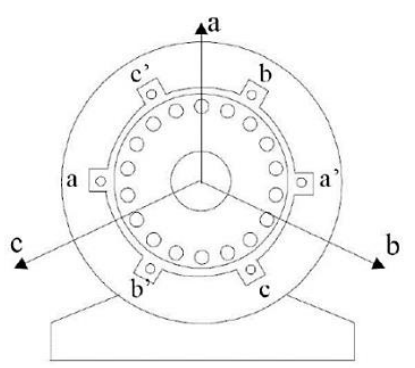

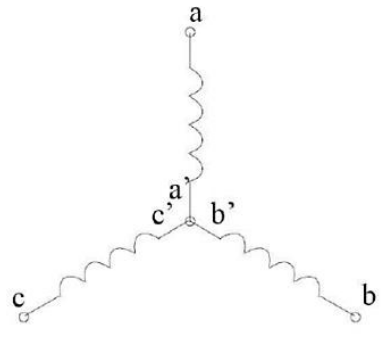

b)

Figure 1. A schematic representation of an asynchronous motor with 6 grooves in the stator and a squirrel-cage type rotor [26].

In the squirrel-cage type rotor, the cage bars and connecting rings are made of aluminum castings for small to mediumpower motors and brass or copper for high power [1]. Regarding the manufacturing type material for the cage, for high efficiency motors, the preferred material for the construction of the cage is copper, mainly due to the higher conductive current density [26].

The stator winding consists of three $2 \pi / 3$ [rad] offset windings in space and P pole pairs. By introducing currents from a three-phase network frequency $f_{1}$, a rotary wave of magnetic force distributed sinusoidal form is produced by the periphery of the air gap, this in turn produces a rotating flow whose velocity is expressed by:

$$
n_{s}=\frac{60 * f_{1}}{P}
$$

where, $\mathrm{n}_{\mathrm{s}}$ is the synchronous speed. This magnetic rotating field will induce an electromagnetic force in the rotor conductors and if its electric circuit is closed, intensities will appear that will try to oppose the variation of stator flux. If, as a limiting case, the rotor rotated at the synchronous speed $n_{s}$, then there would be no movement of the rotating field relative to the rotor, thereby causing the induced electromagnetic force to disappear and as a consequence, current and torque would be canceled out. In this way, the synchronous speed is the theoretical limit at which the rotor can rotate.

The rotor must rotate at a speed lower than the synchronous speed $\left(n<n_{s}\right)$. The difference between the synchronous speed and rotor speed is known by the name of sliding, this value could be between 2-8\% of full load [26]:

$$
s=\frac{\boldsymbol{n}_{s}-\boldsymbol{n}_{r}}{\boldsymbol{n}_{s}} * 100[\%]
$$

As the mechanical load of the motor increases, the resistant torque becomes stronger than the internal torque and the slip increases, causing an increase in the rotor currents, which increases the torque and establishes the dynamic balance of the moments resistant and motor. The frequencies of the rotor currents $f_{2}$ are related to the frequency of the stator by means of the expression:

$$
f_{2}=s * f_{1}
$$

The main faults in IMs can be classified as follow [27]: a). electrical failures: faults in windings due to one or more open circuit or short circuited turns, b). mechanical failures: broken bars, broken slips rings, c) dynamic and static eccentricities, d) curved shaft (dynamic eccentricity), e) failures in bearings.

\section{Variable Frequency Drive (VFD)}

It was showed that the Variable Frequency Drive introduces background noise in the measurements signals, see Figure 3. Therefore, a detail description of the VFD is made in this section. The unwanted noise is both described and reduced. The most widespread method for speed regulation is the use of the VFD. The use of VFD in IMs have been increasing 
continuously, due to their advantages, such as, smooth acceleration and deceleration time, smooth motor starting, increase power factor and last but certainly not least savings cost.

Figure 2 shows the basic operation of a VFD. Stage one rectifies the incoming AC voltage, normally $50 \mathrm{~Hz}$. This is carried out through a non-controlled bridge rectifier. Stage two hold the DC voltage after rectification and filters it. Finally stage three inverts the DC voltage into an AC voltage with controlled frequency.

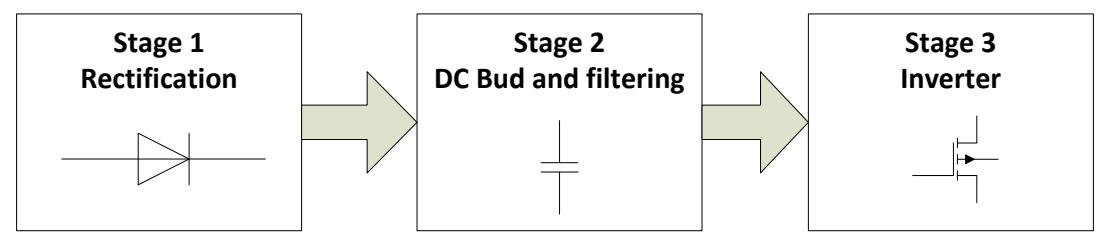

Figure 2. Block diagram of a basic operation VFD.

\section{Effects of the Variable Frequency Drive in Machine Fault Diagnosis}

The use of VFD gives rise to new challenges and drawbacks, which need further research to be overcome. It leads to a question concerning electrical harmonic distortion difficulties. It is important to be aware of, how harmonics are created and the conditions under which harmonics and inter-harmonics could be harmful. It generates also random, high noise levels in the signal, which makes difficult to observe signatures characteristic of motor faults. The characteristics of the noise level will depend on the type of converter, manufacturer, modulation strategy, and control type procedure. Figure 3 illustrates the VFD generation of harmonics and inter-harmonics in all the frequency bands [7], make difficult to observe the actual fault signatures. These inter-harmonics can have higher amplitudes and mask the fault amplitude indicators making it very difficult to distinguish, specially, in the case of incipient faulty signals. To handle and reduce the effect of the inter-harmonics and random noise in the measured raw data. We have designed an anti-aliasing filter, see further details in section Data Acquisition System Design.

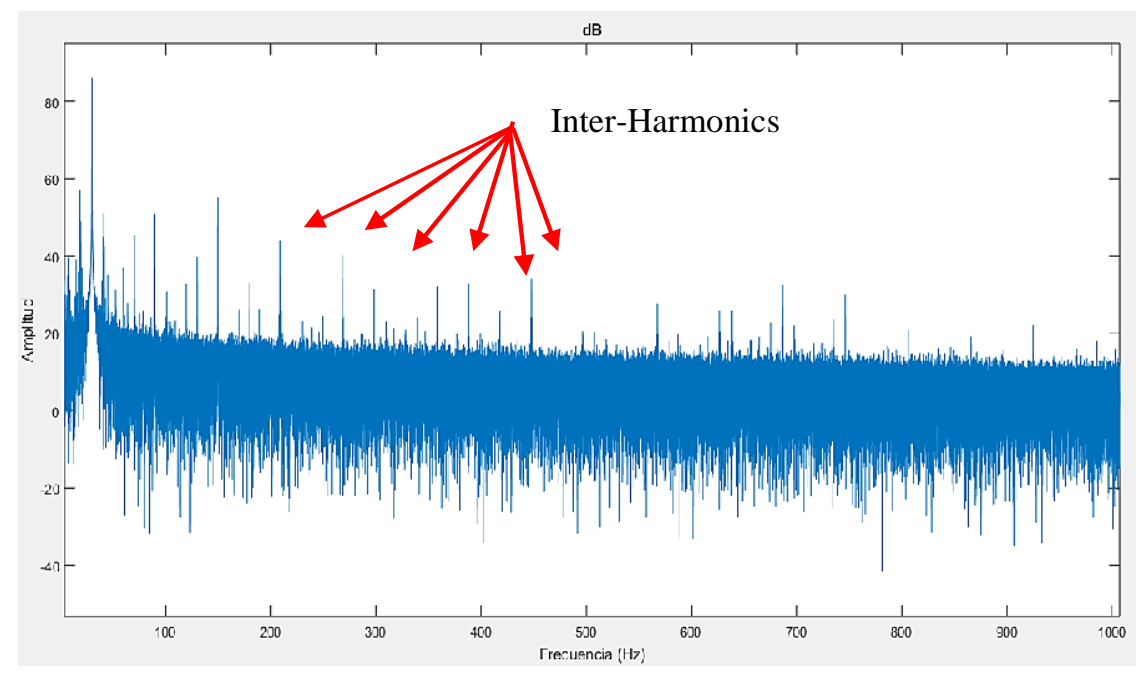

Figure 3. VFD's current spectrum generating noise and inter-harmonics.

\section{Fundamentals of Motor Current Signature Analysis (MCSA) and Digital Signal Processing Techniques}

The MCSA and advanced digital signal processing are reviewed in this section. MCSA is one of the most common methods for diagnosing motor failures because of its low cost and simplicity. The IMs fault generate specific frequency components in the current results, which contains harmonics with potential information of the type of the motor fault. The method is based on the detection of sidebands around the fundamental frequency in the stator current. The MCSA technique only needs the measured current stator data to be processed later and identify small variations in current signatures. This technique provides direct information about the presence of abnormal conditions in electrical machines, the quantification of the failure severity has been an open problem up to date. 


\section{Fast Fourier Transform}

Discrete Fourier transform (DFT) is used to transform data from time to frequency domain. Equation (4) can be used to transform the signal data from time to frequency domain and vice-versa [28] :

$$
X(k)=\sum_{n=0}^{N-1} x(n) e^{\left(\frac{-j 2 \pi k n}{N}\right)}, k=0,1,2, \ldots . . N-1
$$

where, $\mathrm{N}$ is the number of recorded samples of $x(n)$. In the present work, the current signals are transferred from the time domain to the frequency domain using the Fast Fourier transform to further analysis.

\section{The Hilbert Transform (HT)}

The HT is a signal analysis technique used in several areas, one of which is the detection of faults in induction motors. This method is suitable to handle non-stationary and non-linear signals. Mathematically it is defined as the convolution with the function $1 / \pi \mathrm{t}$, as expressed in Eq. (5).

$$
x(t)=\frac{1}{\pi t} * x(t)=\frac{1}{\pi} \int_{-\infty}^{\infty} \frac{x(t)}{t-\tau} d \tau
$$

Summing up the signal $\mathrm{x}(\mathrm{t})$ and the Hilbert Transform it generates the analytical signal $\overrightarrow{x(t)}[10]$ :

$$
\overrightarrow{x(t)}=x(t)+j y(t)=a(t) e^{j \theta(t)} d \tau
$$

where:

$$
\begin{gathered}
a(t)=\sqrt{x^{2}(t)+y^{2}(t)} \\
\theta(t)=\operatorname{artan}\left(\frac{x(t)}{x(t)}\right)
\end{gathered}
$$

The three properties of the Hilbert Transform and the analytical signal most important for signal analysis are: The Hilbert transform of a trigonometric function is the same function shifted $90^{\circ}$. Thus, the spectrum of a Hilbert Transform has the same amplitude and frequency components as the original signal, but the phase of each frequency component is shifted $90^{\circ}$. The analytical signal contains the positive frequency components, while eliminating the negative frequency components and doubles the DC component. All the low frequencies of the original signal are in the analytical amplitude $(\mathrm{t})$, while the high frequencies are in the phase $\theta(\mathrm{t})$.

\section{Envelope Analysis}

Envelope detection is the FFT demodulated signal frequency spectrum of the modulating signal, which shown a high potential to detect incipient faults in machines [13-29]. This method is efficient to detect faults, when the signals, contained near periodic frequency burst, which generates high frequency content for repetitive impulse forces. The envelope of the signal is obtained by using the Hilbert Transform (analytical signal). This is used because it increases the resolution in both frequency and amplitude. This technique proved to be more sensitive under low load conditions. Thus, Abd Elmalek et al. [13] stated that the method presented not only detects whether or not the failure occurs, but it can also determine the number of broken bars and determines the severity of the failure.

\section{SYSTEM ARCHITECTURE AND DESIGN}

\section{Development Board Selection, Active Antialiasing Filter Design, Firmware Development and Software Development.}

This section describes the overall system architecture and design procedure. Initially, the system requirements were to propose the development of Firmware and related Software. It was also observed some guidance for this work in [30], [31] The noise reduction was also handle by active anti-aliasing filters. For further details, the readers recommend to see $[22]$. 


\section{System Requirements and Development Board Selection}

The system requirements are based on: a) low-cost, b) connectivity to internet and network (LAN), c) developed measurement system comparable to standard system, d) flexibility to attach more features. The FRDM K64F developing board, from NXP, was selected as the most appropriate for satisfying the system conditions to collect induction motor`s current data. A complete schematic diagram of the developed system can be seen in Figure 4, which was divided in two sections: a) The analog and b) the digital sections, respectively. The first part, transduces the current measured data, perform signal condition, and it applies the antialiasing filter. The digital part, converts the analog to digital signal and encoded it. The data is capturing in a TCP segment, which is suitable for data transmission over a network. TCP is a highly consistent host to host protocol in packet switched computer networks. The specific details of each component can be found in [22].

The features of the development board, which are relevant for the application are as follows with the Analog and the digital part, respectively.

Analog part

1) Power supply of $5 \mathrm{~V} / 12 \mathrm{~V} / 24 \mathrm{~V} /-12 \mathrm{~V} 120 \mathrm{~W}$

2) Signal conditioner: Sensor signal conditioner model $682 \mathrm{~A} 02$

3) Current sensor

4) Active antialiasing filter built on an instrumentation amplifier that offers a high common-mode rejection ratio (CMRR).

5) Diode clipper circuit limits the voltage range of the signal conditioner output to fit the input voltage ranges of the ADC (0 to $3.3 \mathrm{~V})$ on the Microcontroller Unit.

6) Direct Current (DC) voltage bias circuit adds a DC voltage bias to the output of the signal conditioner to fit the input voltage ranges of the MCU integrated ADC.

Digital part:

1) Development board a MCU with an ARM Cortex-M4 processor which works at $120 \mathrm{MHz}$. Two 16-bit high-speed ADCs with configurable resolution

2) A Micro SD card socket

3) Computer - Machine fault diagnosis.

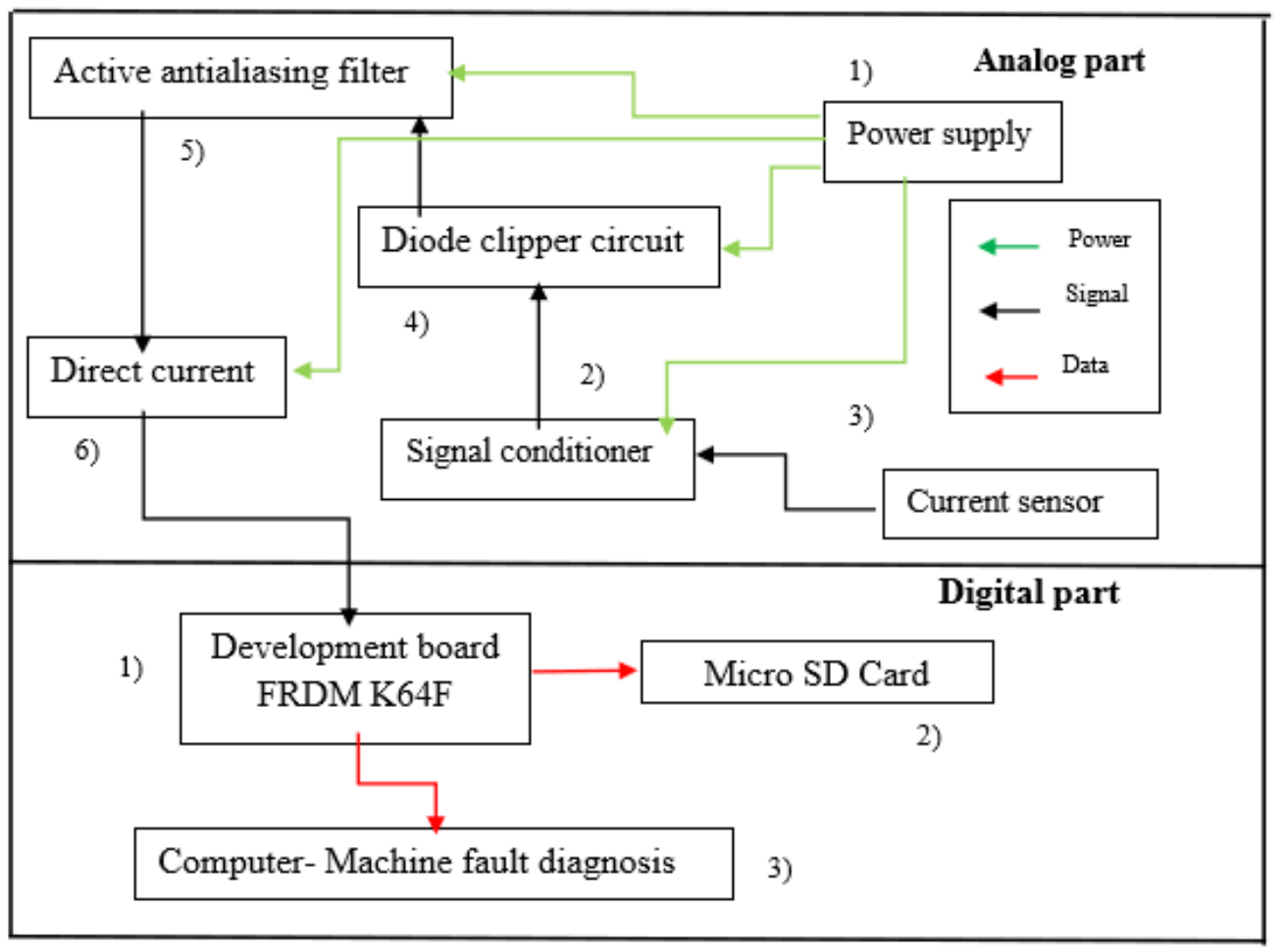

Figure 4. Global schematic developed measurement system for current data analysis.

\section{The Active Anti-Aliasing Filter Design and Firmware Development}

Due to harsh noisy industrial environment, where the developed measurement data acquisition equipment will be used. It has to be considered the Common Mode Rejection Ratio (CMRR). The peculiarity of this filter is an active anti- 
aliasing filter built on an instrumentation amplifier that offers a high CMRR. It is used as a metric to quantify the ability of the device to reject common mode signals, i.e, electromagnetic interference (EMI) signals. Based on aforementioned conditions, therefore, an instrumentation approach, was considered as a second low pass filter based on Blonqvist's [32] . The filters designing parameters are: resistance $10 \mathrm{Kohm}$, and capacitances of $560 \mathrm{pF}$. The cut -off frequency was about $10.6 \mathrm{kHz}$, with $3 \mathrm{~dB}$ decay. The Firmware development was based on the manufacturer's manual (NXP), to choose the selected modules and writing codes. To provide the functionality required by the application, five modules were configured: Multipurpose Clock Generator (MCG), the General Purpose Input Output (GPIO), the Analog/Digital Converter (ADC), the 10/100-Mbps Ethernet MAC (ENET) and the Secured Digital Host Controller (SDHC). We also implemented a TCP client on the board using the middleware lwIP (light weight IP). The MCG was configured to achieve $120 \mathrm{MHz}$ using the Frequency-Locked Loop (FLL). The GPIO ports were configured with their corresponding alternate functions to handle the SD card, the Ethernet interface and the ADC. The ADC was configured in continuous high-speed mode, with 12-bits of resolution and a sampling frequency of $19348 \mathrm{KHz}$. The ENET module was configured to achieve a bit rate of $100 \mathrm{Mbps}$. Finally, the SDHC was set to write 5 blocks of 512 bytes at once and read block by block.

\section{Software Development}

The program starts by initializing all the modules with their corresponding configuration, thereafter, the ADC takes 10 seconds of samples, which are written on the SD card in packets of 2560 bytes ( 5 blocks of 512 bytes). The writing process was applied a ping-pong buffer, as an intermediary between the ADC and the SD card. The ping-pong buffer was made of two buffers; one is the storing the samples of the ADC while the other has being read and it has been written on the SD card. When the first buffer is full and the second is empty, they exchange roles. Once the 10 seconds of samples have been written on the SD card, a Transmission Control Protocol (TCP) client is initiated and requests a connection. If a TCP server replies to the request, the program reads a block of the SD card, encapsulates it into a TCP datagram and dispatches it to the server through the TCP client. The execution of the firmware is part of the measurement process which starts with current sensor that is connected to a signal conditioner which sends a voltage signal to the ADC of the board as depicted in Figure 5. Finally, to analyze the data, a simple TCP server was written in Python. This server receives the datagrams, extracts the data from them and it performs the FFT and EA.

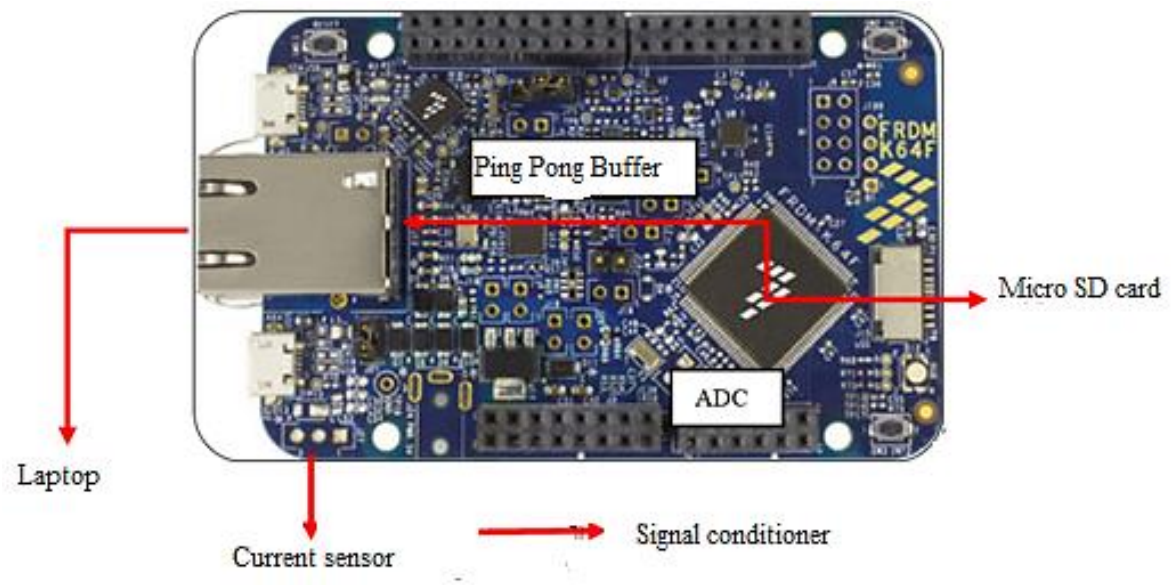

Figure 5. Selected Freescale FRDM-K64F low-cost development board[20] .

\section{MEASUREMENT SET-UP AND PROCEDURE}

An overview of the experimental set-up and the procedure analysis are described in this section. To conduct the experiments, the following sensors and equipment's were used: one motor WEG $1 \mathrm{hp}$, one motor WEG $3 / 4 \mathrm{hp}$, two variable frequency drives WEG CFW100, one tachometer Red Lion IFMA0035, one data acquisition card NI USB-6009, four bearings of type 6204Z, and one PLC Micrologix 1100. The motors are controlled by variable frequency drives of the WEG CFW 100 line, with single-phase 220 VAC input voltage and three-phase output. All the experiments were carried out on the test rig, which can be seen in Figure 6, the new and the standard measurement techniques. To collect the current signal can be performed either using the current sensor or it can be conducted directly from the VDF. Figure 7 denotes the flowchart of the measurement and data analysis procedure with the implemented of the test bench. Stage 1) contains two induction motors mechanically coupled by a flexible coupling. The motor on the left is the one that acts as the actuator, the speed, current, voltage and load signals are monitored. Stage 2) carries out, the data acquisition and data post preprocessing to reduce the noise. 


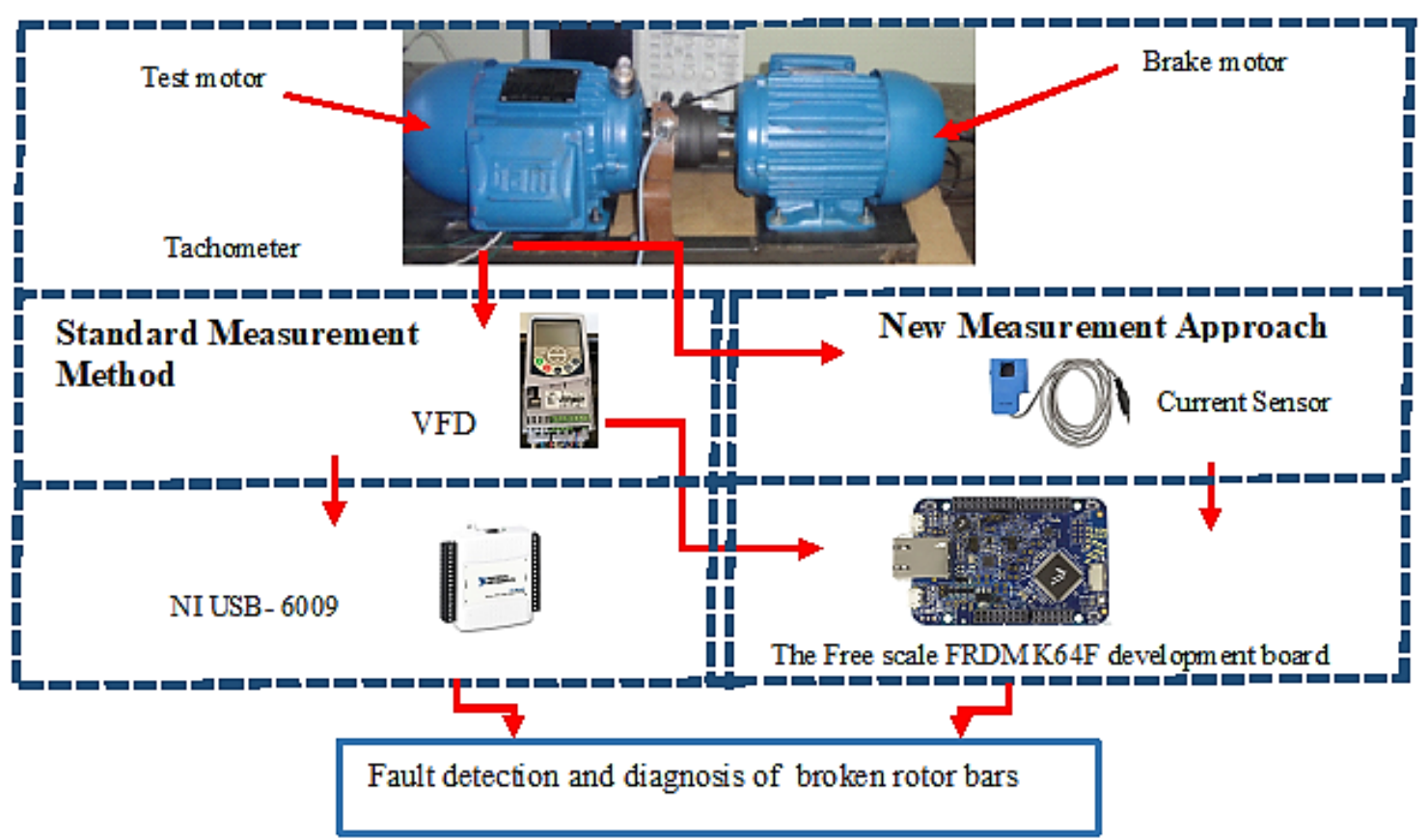

Figure 6. Measurement set-up with the standard and new developed measurement method.

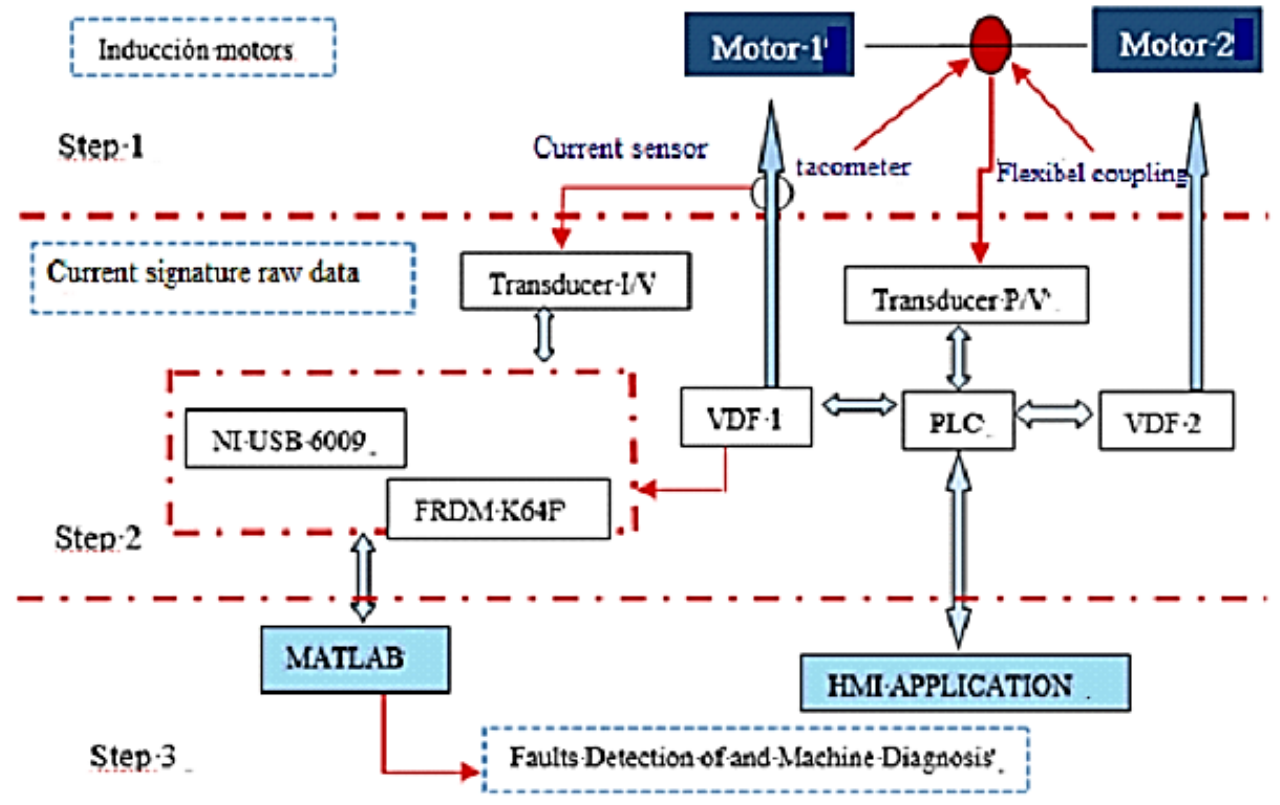

Figure 7. Measurement and analysis procedure.

In order to obtain accurate data, the recommendation is to perform the measurements, only when after the motor reaches steady state, to prevent motor fluctuations in stator current spectrum[13]. To facilitate the control, both the monitoring process and the obtained results were applied the Human Machine Interface (HMI). In Figure 8, the HMI application could display important parameters such as reference frequency, feedback frequency, current, speed, voltage, etc. It provides also a widespread and accurate picture of measurements, operations, maintenance, and production. In our measurement set, both drives, VDF, current signals, voltage and the tachometer are showed in the HMI environment. 


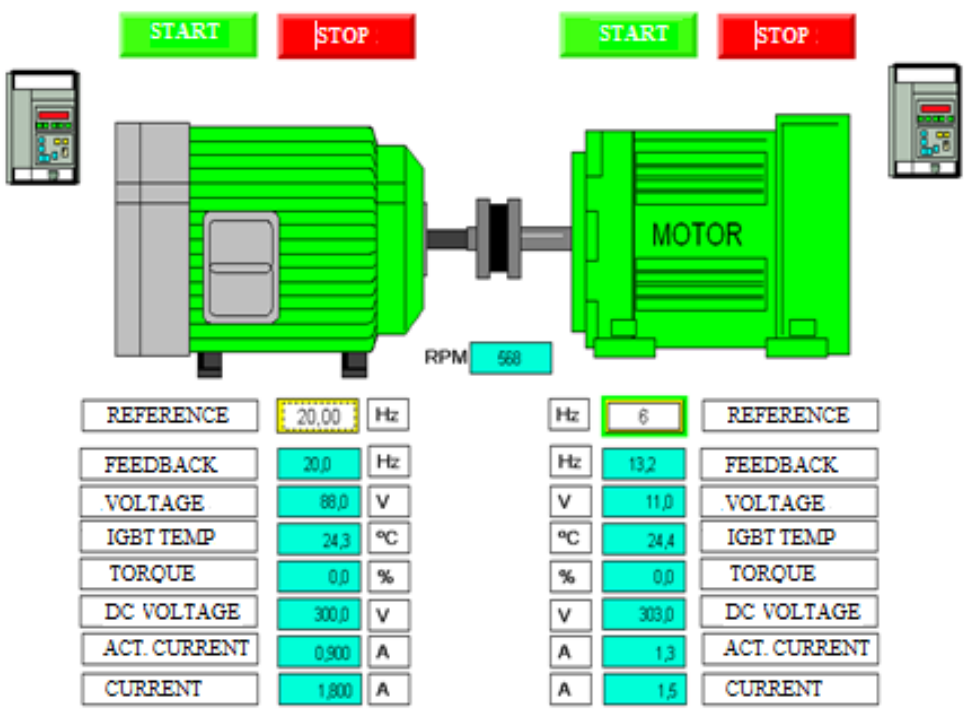

Figure 8. Application of Human Machine Interface (HMI).

\section{Generation of Baseline Measurements and Data Analysis}

It is quite important to establish reference spectrum values to be used for further analysis in machine diagnosis and/or maintenance systems. In order to have a baseline reference signals, measurements with healthy motors, with varying speeds and loads were performed. Thus, it was measured five times for each measurement condition to have more samples data to compare with, and for more details see the experimental plan Table 1. The first set of a healthy induction motor, contains 60 tests and it was performed under varying loads and varying frequencies. In order to reduce speed fluctuation, the measurements were carried out after the motors were stabilized. In the implemented laboratory bench, totally 240 tests were carried out, obtaining current data; for more details, see Table 1, 2 and 3.

Table 1. Number of tests in a healthy induction motor.

\begin{tabular}{cr|cc|cc}
\hline \multicolumn{2}{c|}{ Number of tests at $10[\mathrm{~Hz}]$} & \multicolumn{2}{c}{ Number of tests at $20[\mathrm{~Hz}]$} & \multicolumn{2}{c}{ Number of tests at 30 [Hz] } \\
\hline Load $(\%)$ & $\mathrm{N}^{\circ}$ & Load $(\%)$ & $\mathrm{N}^{\circ}$ & Load $(\%)$ & $\mathrm{N}^{\circ}$ \\
None & 5 & None & 5 & None & 5 \\
28 & 5 & 28 & 5 & 22 & 5 \\
53 & 5 & 40 & 5 & 38 & 5 \\
84 & 5 & 70 & 5 & 48 & 5 \\
\hline \multicolumn{2}{l}{ TOTAL NUMBER OF TESTS }
\end{tabular}

Figure 9 denotes a typical reference healthy motor spectrum of an acquired signal with a line frequency of $30 \mathrm{~Hz}$. It can be stressed out that there are no sidebands around the line frequency.

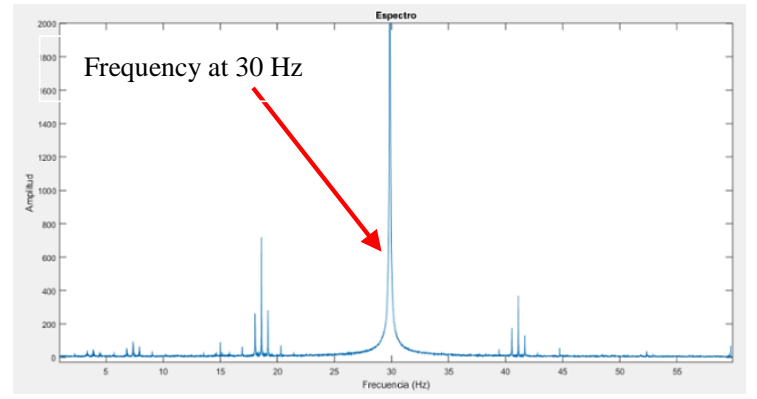

Figure 9. Current signature spectrum signal with no faults at $30 \mathrm{~Hz}$.

Figure 10 illustrates the results of line frequency of $30 \mathrm{~Hz}$, with varying loads $(0 \%, 22 \%, 38 \%$ and $48 \%)$. It can be seen, as expected, no side bands around the line frequency in healthy motors. 


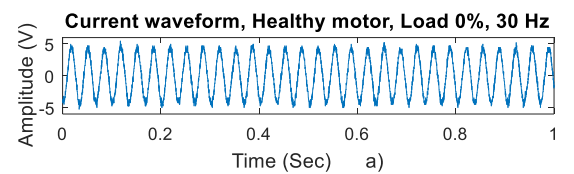

Current waveform, Healthy motor, Load $22 \%, 30 \mathrm{~Hz}$

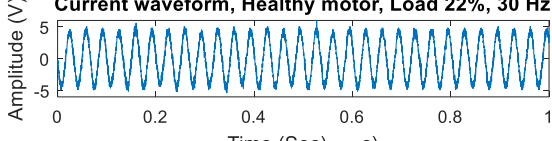

Time (Sec) c)

$\sum$ Current waveform, Healthy motor, Load $38 \%, 30 \mathrm{~Hz}$

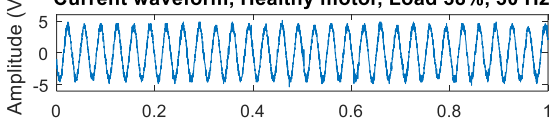

Time (Sec) e)

Current waveform, Healthy motor, Load $48 \%, 30 \mathrm{~Hz}$

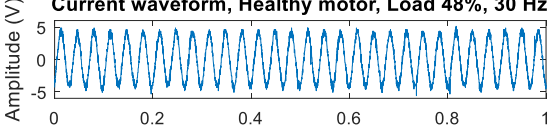

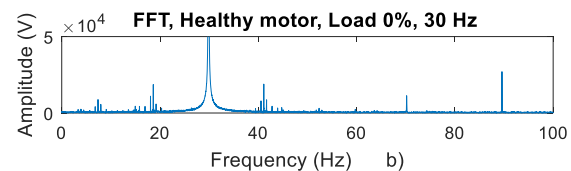
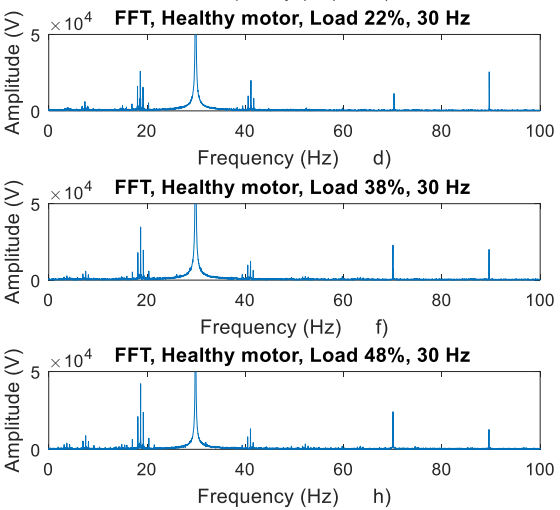

Figure 10. FFT analysis of current signature signals, healthy motor with varying loads and constant speed of $30 \mathrm{~Hz}$.

\section{Generation of Mechanical Damage in Rotors}

The induction motor's magnetic field can be changed, due to machine damage, depending of the failure type, could be more or less important and will affect normal IM performance. If the rotor has a failure, such as, a broken rotor bar or broken slip ring, there will be an effect on the stator. Some reasons to rotor bar broken, according [12]. It can be caused partly by poor operating conditions with high temperatures and due to high mechanical loading, particularly, at the beginning. It can also be due to manufacturing process, poor jointing and defective casting can also generate rotor broken bars. To observe how current spectrums changes with rotor varying running condition. Firstly, the motor failures are mechanically caused, partly in the rotor bar with varying type of severities. Three severity faults levels mechanically generated can be seen in Figure 11. Figure 11 a) shows a broken bar, Figure 11 b) illustrates two broken bars and Figure $11 \mathrm{c})$ shows three broken bars, respectively. The rotor faults were progressively implemented to perform the experiments.
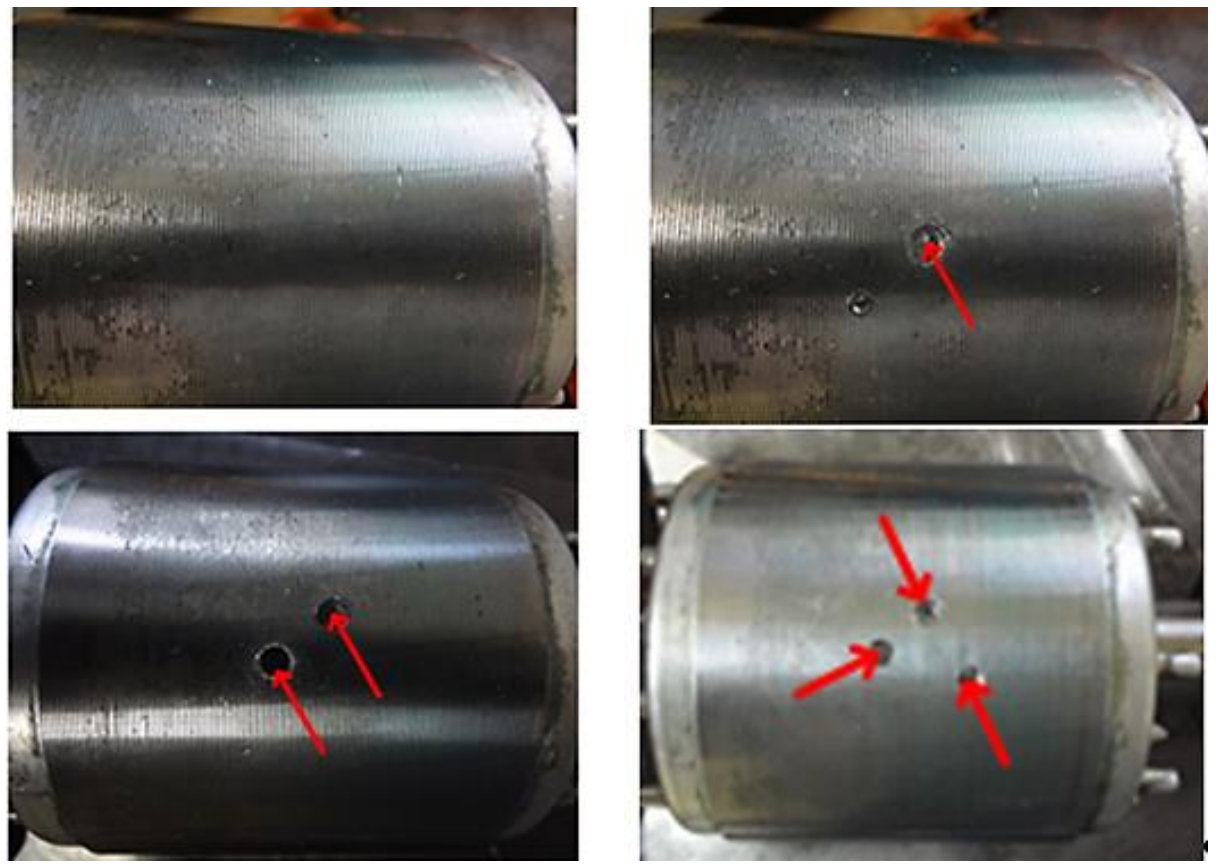

Figure 11. a) Healthy rotor, b) One broken rotor bar (SEV1), b) Two broken rotor bars (SEV2), c) Three broken rotor bars (SEV3). 
Table 2 summarizes the experimental plan of the second measurement data set performed with broken rotor bars. The tests were obtained under varying load conditions, three different operating frequencies $(10,20 \mathrm{and} 30 \mathrm{~Hz})$ and three different severity levels (SEV1 / SEV2 / SEV3).

Table 2. The experimental plan of the second measurement data set.

\begin{tabular}{cccc|ccccc|ccc}
\hline \multicolumn{3}{c|}{ Tests at 10 [Hz] } & \multicolumn{3}{c|}{ Tests at 20 [Hz] } & \multicolumn{3}{c}{ Tests at 30 [Hz] } \\
\hline Load \% & SEV1 & SEV2 & SEV3 & Load \% & SEV1 & SEV2 & SEV3 & Load \% & SEV1 & SEV2 & SEV3 \\
\hline None & 5 & 5 & 5 & None & 5 & 5 & 5 & None & 5 & 5 & 5 \\
28 & 5 & 5 & 5 & 28 & 5 & 5 & 5 & 22 & 5 & 5 & 5 \\
53 & 5 & 5 & 5 & 40 & 5 & 5 & 5 & 38 & 5 & 5 & 5 \\
84 & 5 & 5 & 5 & 70 & 5 & 5 & 5 & 48 & 5 & 5 & 5 \\
\hline
\end{tabular}

\section{RESULTS AND DISCUSSION}

\section{Detection of Broken Rotor Bars by Using MCSA, FFT and Envelope Analysis.}

Three methods, MCSA, FFT and envelope analysis are implemented to diagnose IM broken rotor bar faults. As it was said before, the generation of side bands in the spectrums are due to the effects of broken rotor bar faults. To define its severity, the increasing number of defective rotors bars, increases the signal current amplitudes. Equation (9) defines the sidebands frequency [13].

$$
f_{b}=f_{1} *(1+/ 2 * k * s), k=1,2,3
$$

where $\mathrm{f}_{\mathrm{b}}$ is the sideband frequency, $s$ is the unit slip. The left hand sideband $f_{b}=f_{1} *(1-2 * k * s)$ is due to magnetic rotor asymmetry caused by broken rotor bar, and the amplitudes are direct related to damage severity of the rotor bars. The right hand sideband $f_{b}=f_{1} *(1+2 * k * s)$ is due to speed oscillation due to loading effect [15].

Figure 12 shows the FFT analysis with and without broken rotor bar with $48 \%$ load and speed of $30 \mathrm{~Hz}$. The side bands around the fundamental frequencies are characterized by $27.3 \mathrm{~Hz}$ and $32.4 \mathrm{~Hz}$, respectively. It can also be observed in Figure 13 the summarize results of FFT analysis for different load levels, varying severity levels and a constant line frequency $(20 \mathrm{~Hz})$. The arrows in the Figure 13 shows clearly increasing damage severity, means increasing amplitudes of sidebands.

Hereby, due to this research work it was found out a new consistent and robust parameter (pole pass frequency, $\mathrm{f}_{\mathrm{psf}}$ ), that it could be used as a diagnosis parameter. Figure 14 illustrates, a typical example, a broken rotor bar severity, with $40 \%$ load and speed of $30 \mathrm{~Hz}$. As it can also be seen in the lower graph, the envelope analysis shows the fault characteristic in $2.52 \mathrm{~Hz}$, which corresponds to the pole pass frequency due to one broken bar, see Eq. (11). The Eq. (10) shows the slide value:

$$
\begin{gathered}
s=\frac{\boldsymbol{n}_{s}-\boldsymbol{n}_{r}}{\boldsymbol{n}_{s}}=\frac{900-862}{900}=0.042 \\
f_{p s f}=2 * s^{*} f_{1}=2 * 0.042 * 30=2.52 \mathrm{H}
\end{gathered}
$$



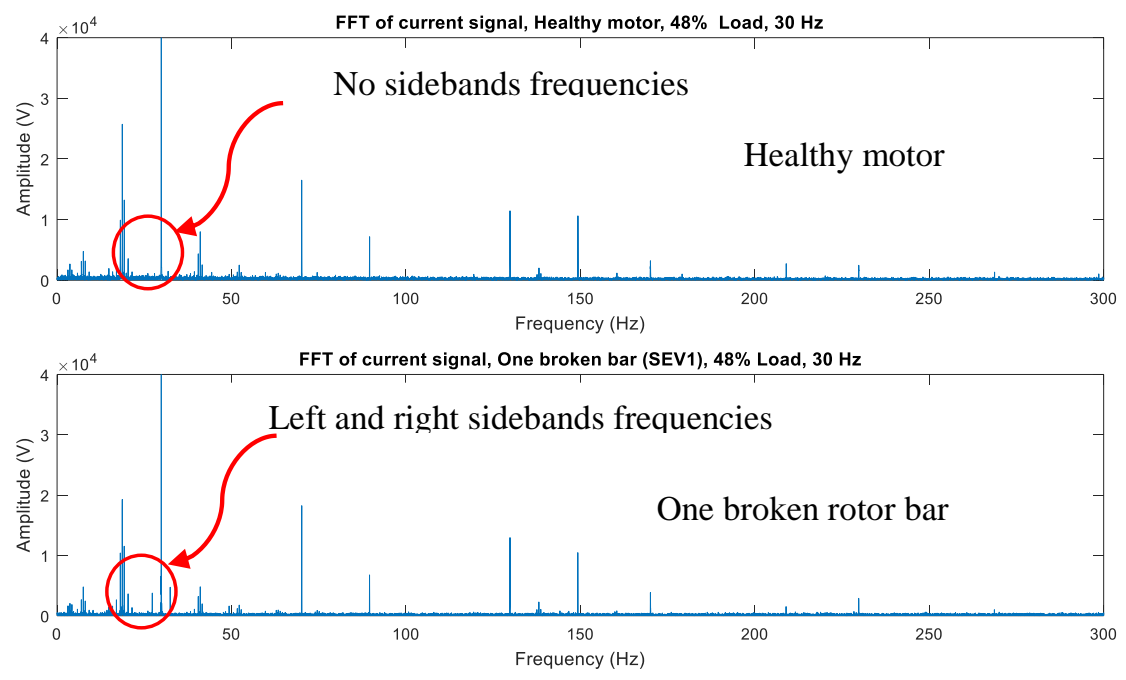

Figure 12. FFT analysis with a healthy motor a) and with broken rotor bar, $48 \%$ load and speed $30 \mathrm{~Hz}$ with severity SEV1 b).
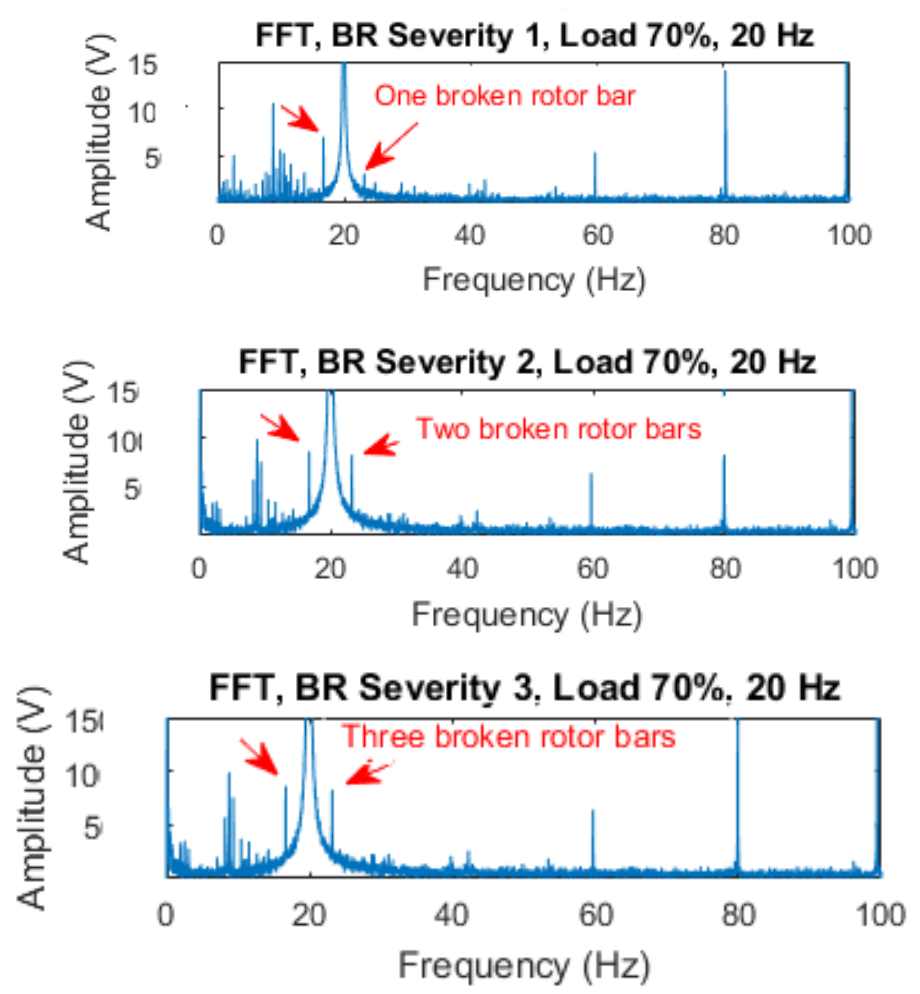

Figure 13. Broken rotor bars analyzed by FFT spectrum with varying severity (SEV1, SEV2, and SEV3) and a constant speed of $20 \mathrm{~Hz}$. 

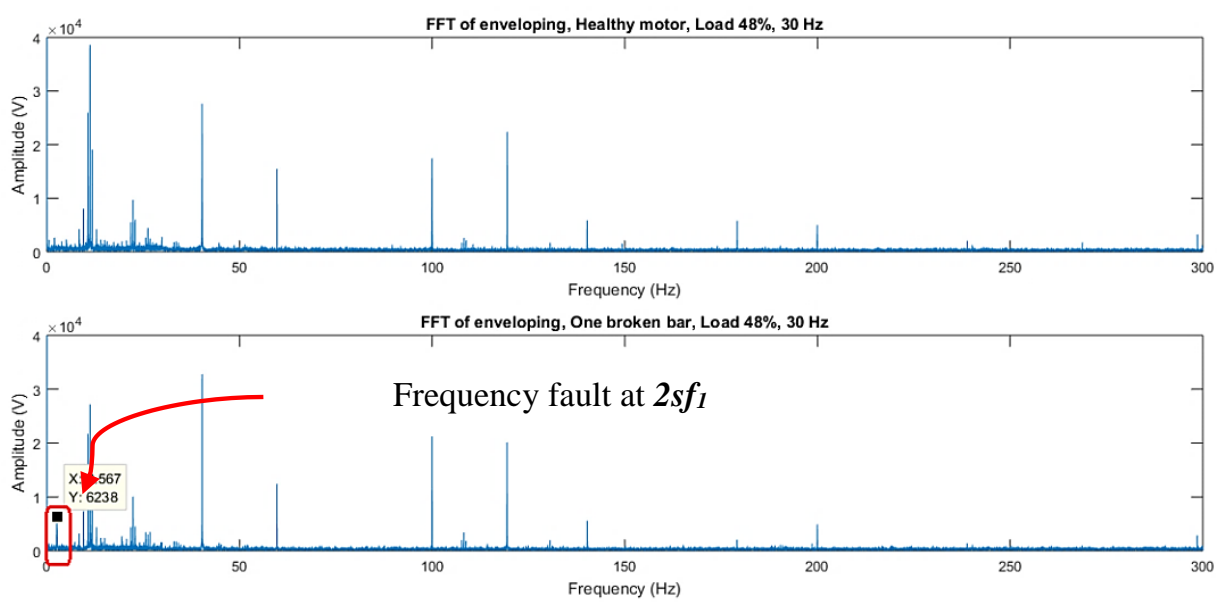

Figure 14. Envelope analysis of broken rotor bar with $48 \%$ Load and speed of $30 \mathrm{~Hz}$.

Figure 15 illustrates the results obtained in the summarized form, with increasing pass pole frequency amplitudes (See the red arrows), due to increasing loads and number of broken rotor bars. It shows the results with one broken rotor bar, two broken rotor bars and three broken rotor bars, respectively. The pass pole frequency amplitudes are around $2.6 \mathrm{~Hz}$.
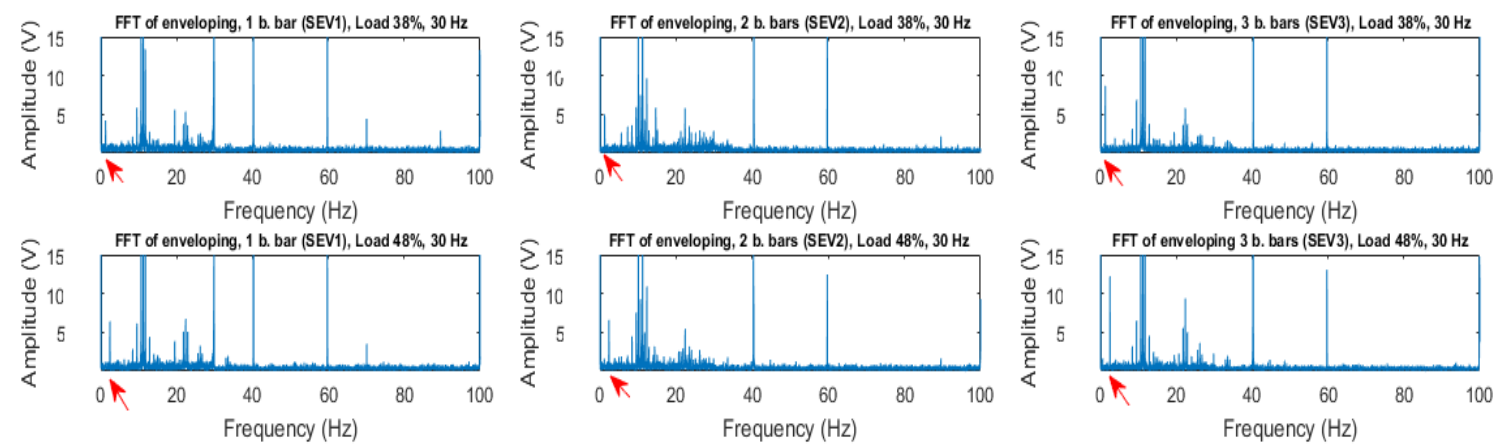

Figure 15. Broken rotor bars analyzed by the enveloped analysis with varying loads (38, \& $48 \%$ Loads) and a constant speed of $30 \mathrm{~Hz}$.

\section{Validation Process: Comparison of Specifications between the Developed and Standard System Measurement System}

The aim of this section is to compare the Frequency Response Function (FRF) of both the developed and the standard measurement systems. The analog simulation circuit of the developed data acquisition system was carried out by Proteous software, see Figure 16 for details.

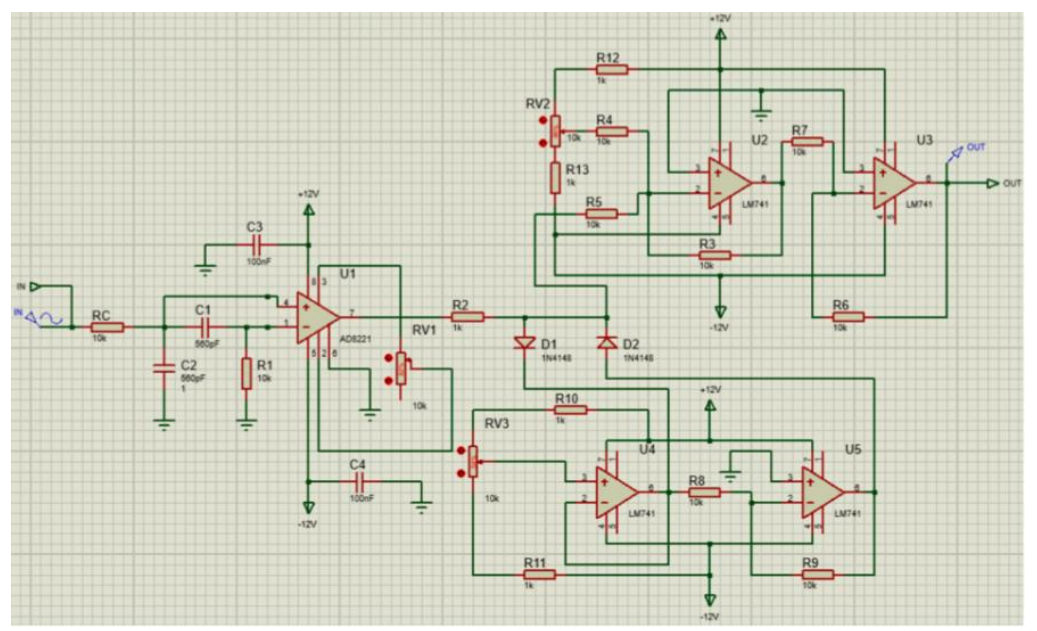

Figure 16. Analog simulation circuit of the developed data acquisition system. 
Figure 17 illustrates, a) Simulated FRF from Proteous software, and b) Experimental FRF of the developed data acquisition measurement system. It can be observed that both results showed constant gain up to our frequency of interest band up to $1 \mathrm{kHz}$.
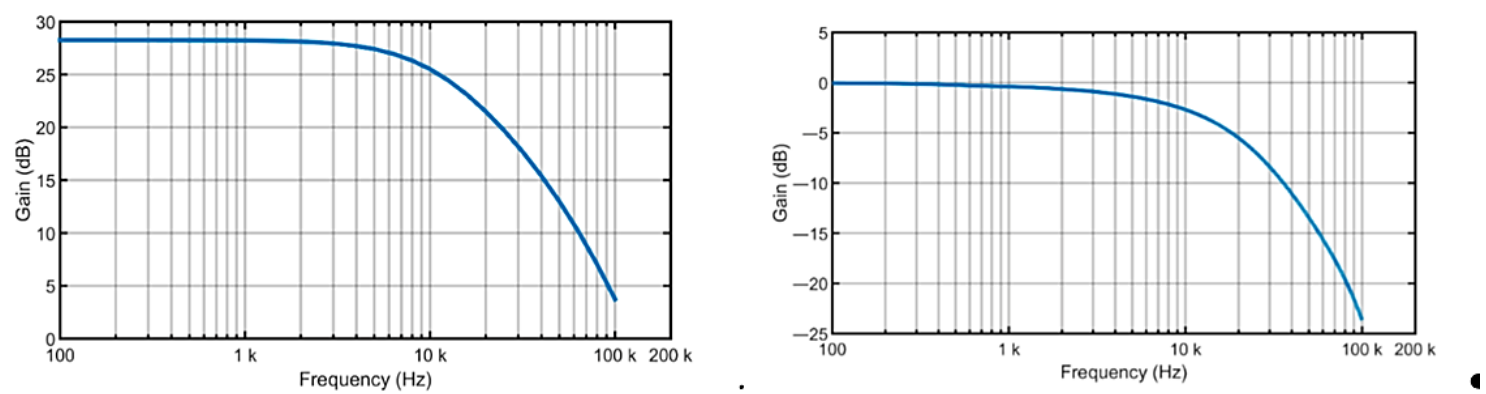

Figure 17. a) Simulated FRF from Proteous software, b) Experimental FRF of the developed data acquisition measurement system.

Table 3 illustrates the obtained values both of the developed system and the standard system (NI 6009) gains, respectively. Our region of frequency interest in this research work is up to $1 \mathrm{kHz}$. It showed a flat frequency spectrum gain from 0 to $1 \mathrm{KHz}$, with small fluctuations, about $0.25 \mathrm{~dB}$ of standard deviation.

To validate the developed data acquisition system, additionally, new measurements were performed. The experiments were carried out with varying speed, load and level of severity. Figure 18 shows the results of FFT analysis for constant load, healthy motor and three broken rotor bars (SEV3), with constant line frequency of $30 \mathrm{~Hz}$.

Table 3. The comparison values of the developed system against standard system of the National Instruments (NI 6009).

\begin{tabular}{c|c|c}
\hline Frequency $(\mathrm{Hz})$ & Developed System Gain $(\mathrm{dB})$ & Standard System gain $(\mathrm{dB})$ \\
\hline 100 & -0.042 & 0 \\
$1 \mathrm{k}$ & -0.396 & 0 \\
$10 \mathrm{k}$ & -2.708 & 0 \\
$100 \mathrm{k}$ & -23.67 & 0 \\
\hline
\end{tabular}
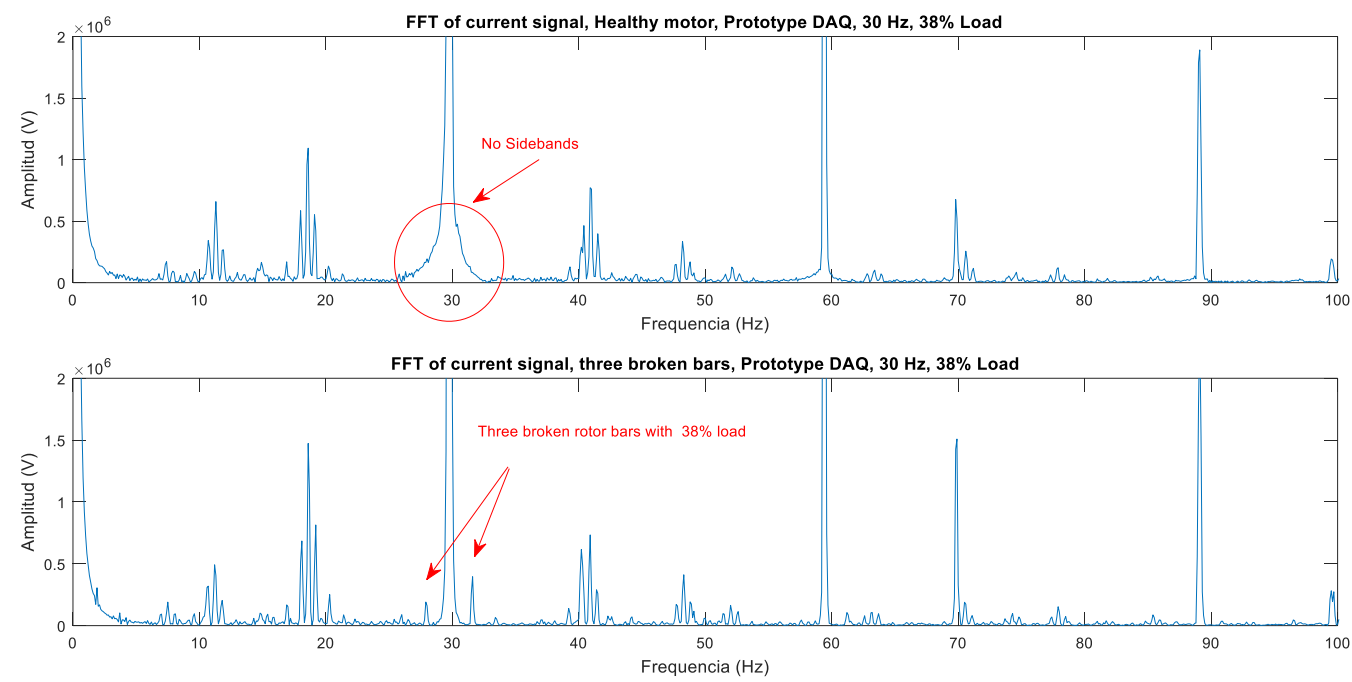

Figure 18. Frequency spectrum of FFT analysis for $30 \mathrm{~Hz}$ and $38 \%$ of load level.

It is clearly noted in Figure 19, increasing loads ( $40 \%$ and $70 \%$ ), mean increasing side bands amplitudes. There are several investigations related to the detection and diagnosis of faults in induction motors using method of current signature analysis $[5,8,11,15,21,23]$, which reveal that there are still some impediments that could degrade performance and accuracy in fault detection and diagnosis. Within given conditions, specially applied to the current signal, the measurements can be sampled only after the IMs reach steady state condition. In other words, the variation of the speed motor could result in fluctuations could give erroneous diagnosis. The above phenomenon is still, an open question for further research. 

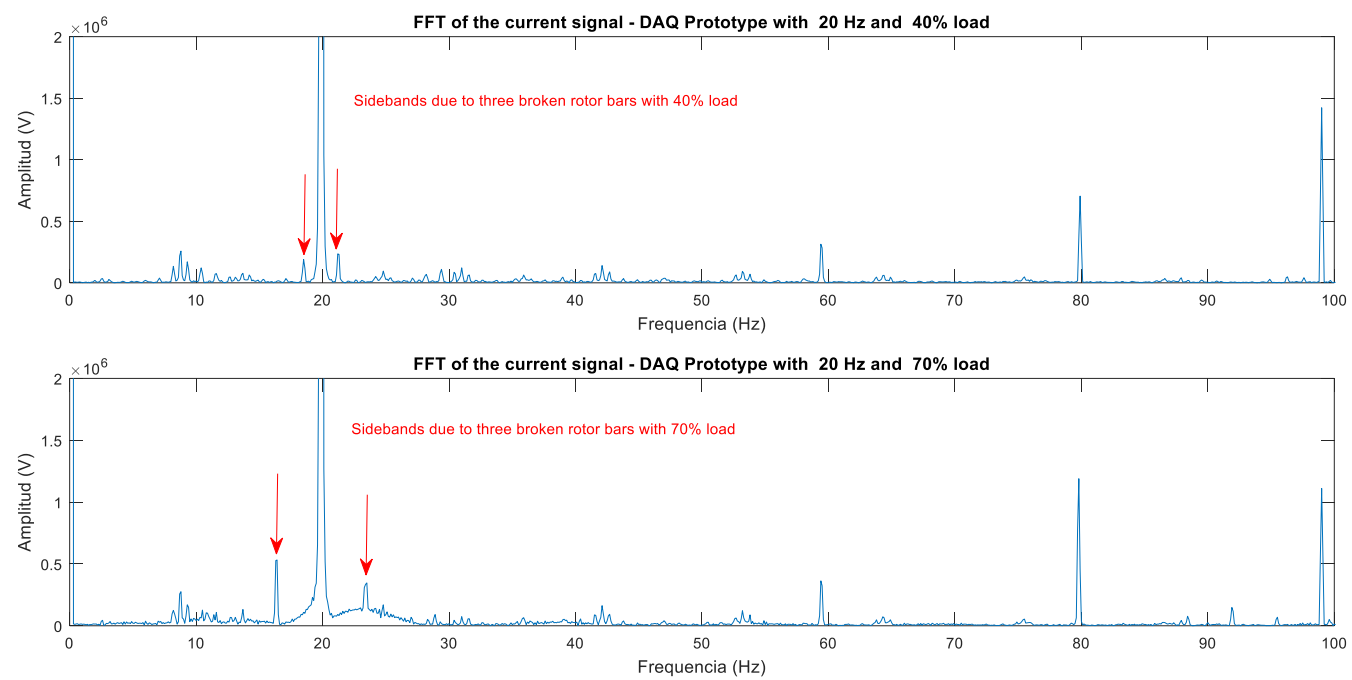

Figure 19. FFT analysis for three broken rotor bars with $20 \mathrm{~Hz}$ and $70 \%$ load level.

\section{CONCLUSION}

The induction motors (IMs) are undoubtedly the most used machines in industries because of the advantages they offer such as simplicity, service continuity and low cost. Due to wear and tear, the motor suffers different types of mechanical and electrical failures. Therefore, in this paper, the research approach was to develop a low cost measurement system based on a micro controller platform for machine diagnosis. The FRDM K64F developing board was selected as the most suitable for satisfying the system conditions, and it was used to collect induction motor`s current data. In order to validate the accuracy of the developed system, the Frequency Transfer Functions(FRF) of the developed measurement system and the standard system (NI USB-6009) were compared. It showed a flat frequency spectrum from 0 to $1 \mathrm{KHz}$, with small fluctuations about $0.25 \mathrm{~dB}$ standard deviation.

A fully automated test bench was implemented, which allowed to perform all the measurement tests with the IMs, and in this case, the detection and diagnosis of broken bars. Around 240 tests were performed, to reduce the effects of the speed fluctuations, with varying loads, different rotation speeds, and with different severity damage levels in the rotor. The data analysis procedure for broken rotor bar detection and motor diagnosis was performed by the Motor Current Signature Analysis (MCSA), FFT and Enveloped Analysis (EA).

Finally, the research approach was successfully accomplished, by developing a software firmware measurement ultralow cost development platform for machine diagnosis. It was also developed a proper antialiasing filter to reduce industrial noise. The effectiveness of the proposed system is detecting a weak fault in a noise signal. It was found out a new consistent and robust parameter called the pole pass frequency $\left(f_{p s f}\right)$, which could be used as a diagnosis parameter for detection of broken rotor bars faults, with their damage severity degree. The detected parameter can be found around $2.6 \mathrm{~Hz}$, and it increases in amplitude with increasing damage severity.

\section{ACKNOWLEDGEMENTS}

The authors would like to express our gratitude to "Universidad Privada Boliviana (UPB)", for all the support to make possible this research project.

\section{REFERENCES}

[1] N. S. Toliya H.A, "Novel frequency domain based technique to detect incipient stator inter-turn faults in induction machines," Ind. Appl. Conf., vol. vol.1, no., 2000.

[2] R. Bond, Vibration-based condition monitoring. Wiley, 2011.

[3] J. Cibulka, M. K. Ebbesen, G. Hovland, K. G. Robbersmyr, and M. R. Hansen, "Review on Approaches for Condition Based Maintenance in Applications with Induction Machines located Offshore," Model. Identif. Control A Nor. Res. Bull., vol. 33, no. 2, pp. 69-86, Sep. 2012, doi: 10.4173/mic.2012.2.4.

[4] A. S. M. Silahuddin, A. M. Aizuddin, S. Mohamaddan, S. T. Syed Shazali, M. S. Z. M. Suffian, A. M. Tazuddin, "Design and development of a modular vibration test rig for combination types of fault in rotating machinery health diagnosis," J. Mech. Eng. Sci., vol. Vol 13. 
[5] P. Shi, Z. Chen, Y. Vagapov, and Z. Zouaoui, "A new diagnosis of broken rotor bar fault extent in three phase squirrel cage induction motor,” Mech. Syst. Signal Process., vol. 42, no. 1-2, pp. 388-403, Jul. 2014, doi: 10.1016/j.ymssp.2013.09.002.

[6] H. H. Kryter, RC., "Electrical signature analysis applications for non-intrusive automotive alternator diagnostics," Meet. Soc. Mach. Fail. Prev. Technol., 1996.

[7] V. F. Pires, M. Kadivonga, J. F. Martins, and A. J. Pires, "Motor square current signature analysis for induction motor rotor diagnosis," Measurement, vol. 46, no. 2, pp. 942-948, Jul. 2013, doi: 10.1016/j.measurement.2012.10.008.

[8] V. Hegde and G. S. Maruthi, "Experimental investigation on detection of air gap eccentricity in induction motors by current and vibration signature analysis using non-invasive sensors," Energy Procedia, vol. 14, pp. 1047-1052, Jul. 2012, doi: 10.1016/j.egypro.2011.12.1053.

[9] A. Roque, J. M. F. Calado, and J. M. Ruiz, "Vibration Analysis versus Current Signature Analysis," IFAC Proc. Vol., vol. 45, no. 20, pp. 794-799, Jul. 2012, doi: 10.3182/20120829-3-MX-2028.00286.

[10] V. Dlamini, R. Naidoo, and M. Manyage, "A non-intrusive method for estimating motor efficiency using vibration signature analysis,” Int. J. Electr. Power Energy Syst., vol. 45, no. 1, pp. 384-390, Jul. 2013, doi: 10.1016/j.ijepes.2012.09.015.

[11] G. Kumar, S. Sharma, and H. Malik, "Learning Vector Quantization Neural Network Based External Fault Diagnosis Model for Three Phase Induction Motor Using Current Signature Analysis," Procedia Comput. Sci., vol. 93, pp. 1010-1016, Jul. 2016, doi: 10.1016/j.procs.2016.07.304.

[12] L. Eren, M. Aşkar, and M. J. Devaney, "Motor current signature analysis via four-channel FIR filter banks," Measurement, vol. 89, pp. 322-327, Jul. 2016, doi: 10.1016/j.measurement.2016.04.025.

[13] M. Abd-el-Malek, A. K. Abdelsalam, and O. E. Hassan, "Induction motor broken rotor bar fault location detection through envelope analysis of start-up current using Hilbert transform," Mech. Syst. Signal Process., vol. 93, pp. 332-350, Jul. 2017, doi: 10.1016/j.ymssp.2017.02.014.

[14] S. Aouabdi, M. Taibi, S. Bouras, and N. Boutasseta, "Using multi-scale entropy and principal component analysis to monitor gears degradation via the motor current signature analysis,” Mech. Syst. Signal Process., vol. 90, pp. 298-316, Jul. 2017, doi: 10.1016/j.ymssp.2016.12.027.

[15] I. Bravo-Imaz, H. Davari Ardakani, Z. Liu, A. García-Arribas, A. Arnaiz, and J. Lee, "Motor current signature analysis for gearbox condition monitoring under transient speeds using wavelet analysis and dual-level time synchronous averaging," Mech. Syst. Signal Process., vol. 94, pp. 73-84, Jul. 2017, doi: 10.1016/j.ymssp.2017.02.011.

[16] P. Bilski and W. Winiecki, "A low-cost real-time virtual spectrum analyzer," IEEE Trans. Instrum. Meas., vol. 56, no. 6, pp. 2169-2174, 2007, doi: 10.1109/TIM.2007.908269.

[17] M. K. Adeyeri, K. Mpofu, and B. Kareem, "Development of hardware system using temperature and vibration maintenance models integration concepts for conventional machines monitoring: a case study," J. Ind. Eng. Int., vol. 12, no. 1, pp. 93-109, 2016, doi: 10.1007/s40092-015-0132-8.

[18] H. F. Zhang and W. Kang, "Design of the data acquisition system based on STM32," Procedia Comput. Sci., vol. 17, pp. 222228, 2013, doi: 10.1016/j.procs.2013.05.030.

[19] G. Huang and Y. Fan, "Design and realization of vibration signal acquisition \& analysis system based on STM32," 2016, pp. 2924-2928, doi: 10.1109/CCDC.2016.7531482.

[20] S. J. Hester J, Prabhu, S, atamturktur S, "Remote and wireless long term vibration monitoring of historic monuments.," Procedia Eng., vol. 168, no. 3302-3307, 2017.

[21] M. F. Herrasti Z, Gabilondo I, Berganzo J, Val I., "Wireless sensor nodes for acceleration strain and temperature measurements," Procedia Eng., pp. 1659-1662, 2016.

[22] A. Villarroel, G. Zurita, and R. Velarde, "Development of a low-cost vibration measurement system for industrial applications," Machines, vol. 7, no. 1, 2019, doi: 10.3390/machines7010012.

[23] E. P. Carden and P. Fanning, "Vibration Based Condition Monitoring: A Review," Struct. Heal. Monit. An Int. J., vol. 3 , no. 4, pp. 355-377, Sep. 2004, doi: 10.1177/1475921704047500.

[24] Z. Dragomir OE, Gouriveau R, Dragomir F, Minca E, "Review of prognostic problem in condition-based maintenance.," in 2009 European Control Conference (ECC). IEEE:, 2009, pp. 1587-1592.

[25] A. Prajapati, J. Bechtel, and S. Ganesan, “Condition based maintenance: a survey,” J. Qual. Maint. Eng., vol. 18, no. 4, pp. 384-400, Sep. 2012, doi: 10.1108/13552511211281552.

[26] H. P. Ruben P, Manuel P, Martin R, Jose F, "Improved resolution of the MCSA method via Hilbert transform. enabling the diagnosis of rotor asymetries at lower slip.," IEEE Trans. Energy Convers. 2009; , vol. 24, pp. 52-59, 2009.

[27] H. H. Kryter R, "Condition monitoring of machinery using motor current signature analysis.," J. Sound Vib., vol. 23, pp. 1421, 1989.

[28] S. O., Digital Signal Processing. Pearson, 2015.

[29] A. B. Ming, W. Zhang, Z. Y. Qin, and F. L. Chu, "Envelope calculation of the multi-component signal and its application to the deterministic component cancellation in bearing fault diagnosis," Mech. Syst. Signal Process., vol. 50-51, pp. 70-100, Jul. 2015, doi: 10.1016/j.ymssp.2014.05.033.

[30] M. A. S. M. Tahir, J. A. Ghani, M. Z. Nuawi, M. Rizal, and C. H. C. Haron, "Flank wear and I-kaz 3D correlation in ball end milling process of Inconel 718," J. Mech. Eng. Sci., vol. 9, no. December, pp. 1595-1603, 2015, doi: 10.15282/jmes.9.2015.7.0155. 
[31] H. M. Rizal1, J.A. Ghani, Husni, "Design and construction of a strain gauge-based dynamometer for a 3-axis cutting force measurement in turning process," J. Mech. Eng. Sci., vol. 12, no. 4, 2018.

[32] S. R. Blomqvist KH, Eskelinen P, "Instrumentation amplifier implements second-order active low-pass filter with high gain factor.," Meas. Sci. Technol., vol. 22, 2011. 